\title{
Characteristics and outcomes of anti-infective de-escalation during health care-associated intra-abdominal infections
}

Philippe Montravers ${ }^{1,2^{*}}$, Pascal Augustin ${ }^{1}$, Nathalie Grall ${ }^{2,3,4}$, Mathieu Desmard ${ }^{1,5}$, Nicolas Allou', Jean-Pierre Marmuse ${ }^{2,6}$ and Jean Guglielminotti, ${ }^{1,3}$

\begin{abstract}
Background: De-escalation is strongly recommended for antibiotic stewardship. No studies have addressed this issue in the context of health care-associated intra-abdominal infections (HCIAI). We analyzed the factors that could interfere with this process and their clinical consequences in intensive care unit (ICU) patients with HCIAl.

Methods: All consecutive patients admitted for the management of HCIAI who survived more than 3 days following their diagnosis, who remained in the ICU for more than 3 days, and who did not undergo early reoperation during the first 3 days were analyzed prospectively in an observational, single-center study in a tertiary care university hospital.

Results: Overall, 311 patients with HCIAI were admitted to the ICU. De-escalation was applied in 110 patients (53\%), and no de-escalation was reported in 96 patients (47\%) (escalation in 65 [32\%] and unchanged regimen in 31 [15\%]). Lower proportions of Enterococcus faecium, nonfermenting Gram-negative bacilli (NFGNB), and multidrugresistant (MDR) strains were cultured in the de-escalation group. No clinical difference was observed at day 7 between patients who were de-escalated and those who were not. Determinants of de-escalation in multivariate analysis were adequate empiric therapy (OR 9.60, $95 \% \mathrm{Cl}$ 4.02-22.97) and empiric use of vancomycin (OR 3.39, $95 \%$ Cl 1.46-7.87), carbapenems (OR 2.64, $95 \% \mathrm{Cl}$ 1.01-6.91), and aminoglycosides (OR $2.3195 \% \mathrm{Cl}$ 1.08-4.94). The presence of NFGNB (OR $0.28,95 \% \mathrm{Cl} 0.09-0.89$ ) and the presence of MDR bacteria (OR $0.21,95 \% \mathrm{Cl} 0.09-0.52$ ) were risk factors for non-de-escalation. De-escalation did not change the overall duration of therapy. The risk factors for death at day 28 were presence of fungi (HR 2.64, $95 \% \mathrm{Cl} 1.34-5.17)$, Sequential Organ Failure Assessment score on admission (HR 1.29, $95 \% \mathrm{Cl} 1.16-1.42)$, and age (HR 1.03, $95 \% \mathrm{Cl}$ 1.01-1.05). The survival rate expressed by a Kaplan-Meier curve was similar between groups (log-rank test $p$ value 0.176).
\end{abstract}

Conclusions: De-escalation is a feasible option in patients with polymicrobial infections such as HCIAl, but MDR organisms and NFGNB limit its implementation.

Keywords: De-escalation, Health care-associated intra-abdominal infections, Peritonitis, Multidrug-resistant bacteria, Antibiotic therapy

\footnotetext{
* Correspondence: philippe.montravers@aphp.fr

${ }^{1}$ Département d'Anesthésie Réanimation, APHP, CHU Bichat-Claude Bernard,

Paris, France

${ }^{2}$ Université Denis Diderot, PRESS Sorbonne Cité, Paris, France

Full list of author information is available at the end of the article
} 


\section{Background}

The modern concept of reducing both the spectrum of antimicrobial therapy and its potential to promote resistance [1], usually called de-escalation, is strongly recommended in all of the recently published guidelines for antibiotic stewardship [2, 3]. Several definitions have been used to describe this process [1, 4-7]. Despite the limited evidence, de-escalation has been recommended to decrease the likelihood of emergence of resistant organisms [8], to optimize activity, and to reduce toxicity and costs [3].

Two types of critically ill patients have been investigated: cohorts with a specific disease-mainly ventilatorassociated pneumonia (VAP) [5, 9-13]-and large mixed populations with severe sepsis or septic shock or patients who require emergency empiric antibiotic therapy [4, 14-19]. In a recent systematic review, Tabah et al. identified isolation of multiresistant pathogens, polymicrobial infections, and intra-abdominal infections as factors negatively associated with de-escalation [20]. Only a few studies have addressed this issue in the context of intraabdominal infections $[4,14,18,21,22]$. The high frequency of polymicrobial infection [23, 24] and multidrugresistant (MDR) organisms [23, 24] as well as the presence of fungi $[17,25]$ in this setting might raise specific concerns, especially in health care-associated intra-abdominal infections (HCIAI).

The purpose of the present study was to analyze the frequency of de-escalation, the factors that could interfere with this process, and their clinical consequences in a cohort of intensive care unit (ICU) patients with HCIAI.

\section{Methods}

\section{Study population}

From January 1999 through December 2011, all consecutive patients admitted to our ICU for the management of HCIAI were prospectively included in a database and their medical charts were retrospectively reviewed. The study was approved by the local institutional review board (CEERB CHU Bichat Paris VII University, APHP, Paris, France), which waived the need for signed informed consent.

\section{Selection of cases and inclusion criteria}

Postoperative peritonitis was defined as the first macroscopic findings of intra-abdominal infection combined with positive fluid culture yielding at least one microorganism (bacteria or fungi) at the time of reoperation (day 0) following a first abdominal surgery [24]. Several patients had to be excluded from the analysis due to early change in their clinical status before de-escalation could be instituted: patients who died during the first 3 days following surgery (microbiologic results not yet obtained), those who were discharged during the first 3 days (incapacity to adequately follow clinical outcome and antibiotic therapy), and those who underwent early reoperation during the first 3 days (high proportion of persistent infection and prolonged antibiotic therapy). Similarly, we excluded patients with negative microbiologic samples, since the concept of de-escalation is questionable and the interpretation of the results is difficult. In these patients, empiric anti-infective therapy was discontinued. Drainage of abscesses, debridement of infected and necrotic tissues, abdominal cavity cleansing, irrigation, and definitive control of the source of contamination were performed according to the surgical principles used for the management of abdominal sepsis [26]. Ostomy was preferred to primary anastomosis. No open-wound management was performed, and the abdomen was not irrigated after surgery.

\section{Microbiologic data}

Peritoneal fluid samples were systematically collected during surgery and were immediately sent to the bacteriology laboratory [24]. Cultures were performed with identification and susceptibility testing for Gram-positive and Gram-negative aerobe bacteria, anaerobes, and fungi. Antibiotic susceptibility was determined for each organism by the disk diffusion method, according to the criteria of the Antibiogram Committee of the French Society for Microbiology [27]. MDR bacteria were defined as those resistant to three or more antimicrobial classes [28]: methicillinresistant Staphylococcus aureus and methicillin-resistant coagulase-negative staphylococci; ampicillin-resistant enterococci; Enterobacteriaceae producing an extendedspectrum $\beta$-lactamase or producing a derepressed cephalosporinase; and/or nonfermenting Gram-negative bacilli (NFGNB) resistant to piperacillin-tazobactam, ceftazidime, or imipenem-cilastatin, or producing an extendedspectrum $\beta$-lactamase.

\section{Management of antibiotic therapy}

Empiric anti-infective therapy, systematically started at day 0 , took into account the severity of the case and usually combined piperacillin-tazobactam or imipenemcilastatin with amikacin and vancomycin [23], possibly associated with antifungal therapy (mainly fluconazole) based on presumed risk factors [25, 29]. Definitive antiinfective therapy was adapted on the basis of the results of identification and antibiotic susceptibility testing ( $\geq 48 \mathrm{~h}$ ). In both situations, therapy was considered appropriate when all cultured organisms (bacteria and fungi) were targeted. Anti-infective therapy was prescribed by the senior ICU physicians following discussion with the consultant microbiologist on a daily basis.

The following changes were considered to constitute de-escalation [1]: withdrawal of one agent $(\beta$-lactam, 
aminoglycoside, fluoroquinolone, vancomycin, antifungal agent) or narrowing spectrum of activity ( $\beta$-lactam agents) and/or switch from combination to monotherapy. Discontinuation of unduly administered agents was also recorded. Changes among cefepime, ceftazidime, piperacillin-tazobactam, and ticarcillin-clavulanate were not considered to be significant changes of the spectrum of coverage [1].

In patients without de-escalation, two situations were identified according to previous definitions [4]. Maintained empiric treatment without modification was called unchanged therapy [4]. Escalation was defined as addition or switch to a new broad-spectrum anti-infective agent (carbapenems, glycopeptides, fluoroquinolones) [4] or upgrade to broader-spectrum $\beta$-lactams [1]. When changes combined escalation and de-escalation, the patient was assigned to the escalation group [4]. In summary, a patient receiving empiric therapy with piperacillin-tazobactam plus amikacin who was subsequently switched to piperacillin and vancomycin was classified as having withdrawal of one agent and escalation.

\section{Data collection}

All patients' charts were reviewed. Demographic data and severity scores (Simplified Acute Physiology Score II score [30] and Sequential Organ Failure Assessment [SOFA] score [31]) were recorded on admission to the ICU. The severity of the underlying medical condition and the presence of chronic diseases [32] were recorded. The characteristics of initial surgery were recorded.

The following clinical and severity characteristics were assessed at day 0 , day 3 , and day 7 after surgery for patients still in the ICU [24, 33, 34]: temperature, white blood cell count (WBC), serum creatinine, and SOFA score. Patients meeting the following three criteria at day 3 were arbitrarily defined as improving: (1) a SOFA score that decreased more than 2 points at day 3 versus day 0 or a SOFA score of 0 points, (2) a WBC that decreased more than $5000 / \mathrm{mm}^{3}$ between day 0 and day 3 or WBC less than $12,500 / \mathrm{mm}^{3}$, and (3) a temperature decrease greater than $0.5{ }^{\circ} \mathrm{C}$ between day 0 and day 3 or temperature greater than or equal to $36.5{ }^{\circ} \mathrm{C}$ and less than $38.1{ }^{\circ} \mathrm{C}$. Similar analyses were used at day 7 to compare changes in these criteria between days 3 and 7 . Medical and surgical complications, additional reoperations for persistence of the initial infection or superinfections (including MDR organisms), death between days 3 and 28 following surgery, and discharge from the hospital were assessed.

\section{Statistical analysis}

Results are expressed as median and interquartile range (IQR) or number and proportion. Statistical significance was defined as $p<0.05$. For statistical analysis, we used
$\mathrm{R}$ version 2.14.1 software (R Foundation for Statistical Computing, Vienna, Austria). For comparisons between antibiotic strategy groups (de-escalation, no change, or escalation), we used the $x^{2}$ test and Fisher's exact test for discrete variables and unpaired Wilcoxon tests for quantitative variables. The effect of antibiotic strategy on day 28 mortality was assessed with a Kaplan-Meier survival curve and tested with a log-rank test.

Three multivariable models were developed (1) to identify risk factors for de-escalation and (2) to assess the association between antibiotic strategy (de-escalation, no change, or escalation) and day 28 or inhospital mortality. In univariate analysis for these three models, we used Fisher's exact tests and Wilcoxon tests. Unadjusted ORs or HRs were calculated. Variables with a $p$ value less than 0.2 in univariate analysis were entered into a multivariate logistic regression model or a Cox proportional hazards model with backward selection. For day 28 and in-hospital mortality, the antibiotic strategy was forced until the end of the selection process. Logistic models were evaluated for discrimination with the c-statistic and for calibration with the HosmerLemeshow test.

\section{Results \\ Epidemiologic and clinical characteristics}

During the study period, 311 ICU patients were admitted for the management of HCIAI. Figure 1 displays a flowchart of patients through the study. Overall, 105 patients were excluded, resulting in 206 patients for whom the de-escalation process was analyzed. De-escalation was performed in 110 patients (53\% of the analyzed population), and no de-escalation was observed in $96 \mathrm{pa}-$ tients $(47 \%)$ (escalation in 65 patients [32\%] and unchanged regimen in 31 patients [15\%]). De-escalation was never performed after discharge from the ICU. The frequency of de-escalation remained stable over the study period, ranging between $47 \%$ and $63 \%$ of the analyzed population (not significant; data not shown). Clinical characteristics were similar at day 0 in both groups (Table 1). In the non-de-escalation group, a significantly increased severity was observed in the patients with an unchanged regimen versus escalation (Table 1).

\section{Microbiologic analysis}

Overall, 618 microorganisms from peritoneal samples were cultured (311 in patients without de-escalation, including 101 organisms in the unchanged group and 210 in the escalation group). Similar microbiologic results were observed between de-escalation and no deescalation groups (data not shown), except for lower proportions of Enterococcus faecium (9 [3\%] versus 18 [7 \%] without de-escalation, respectively; $p<0.01)$ and non-fermenting Gram-negative bacilli (9 [3\%] versus 22 


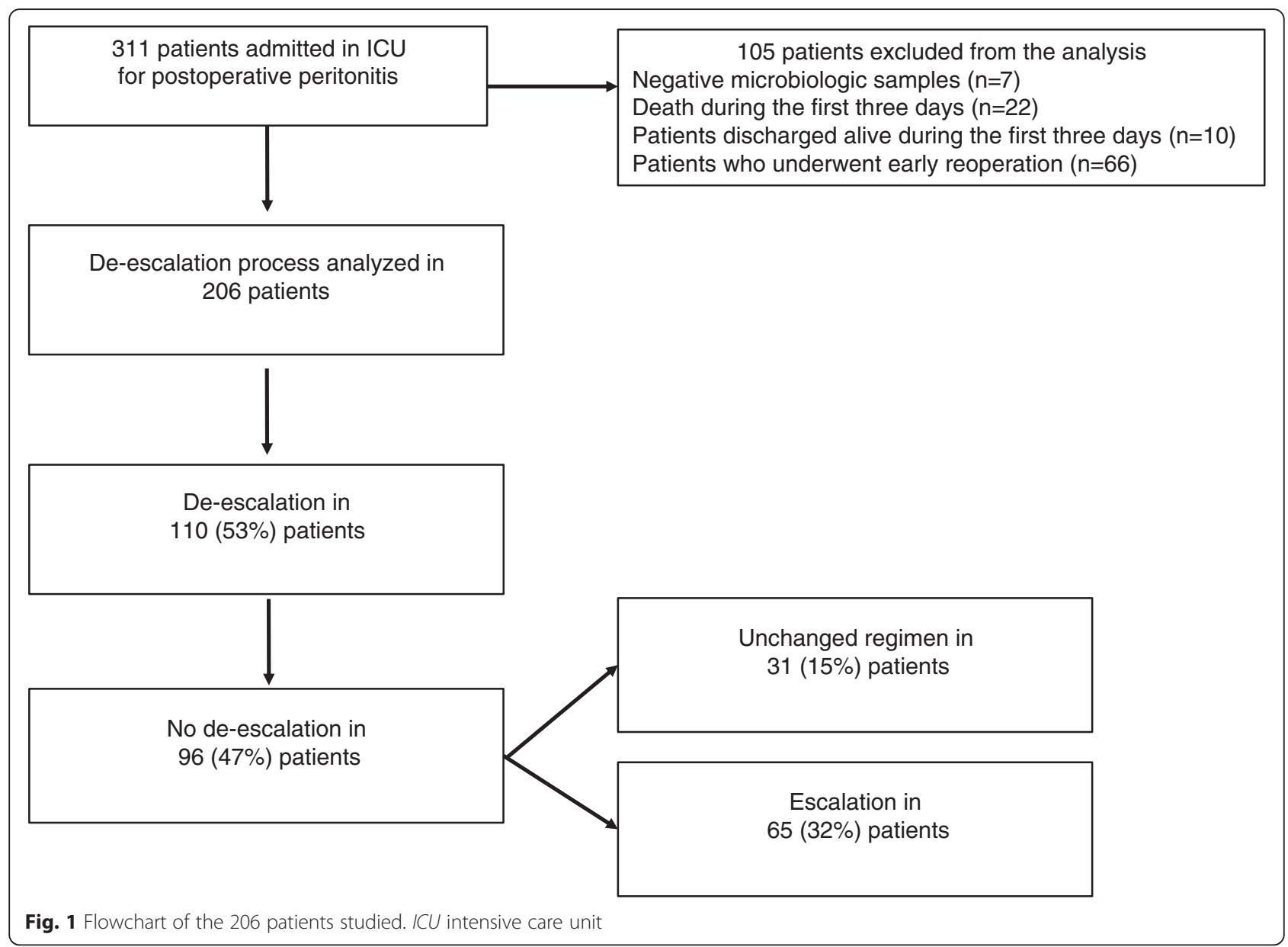

[8\%], respectively; $p<0.01$ ). Among patients without de-escalation, increased proportions of Gram-negative bacteria and Enterobacteriaceae were observed in patients with unchanged regimen compared with those whose regimen was escalated (47 [49 \%] versus 69 [37\%], respectively $[p<0.05]$; and 38 [40\%] versus 52 [28\%] $[p<0.05])$. In the de-escalation group, 23 (21\%) of 110 patients harbored MDR strains compared with $54(56 \%)$ of 96 patients in the non-de-escalation group $(p<0.01)($ Table 2$)$.

\section{Anti-infective therapy}

De-escalation was performed on a microbiologic basis in a median delay of 3 days (IQR 2-4) after surgery. Empiric treatments and the procedures applied for deescalation at day 3 are described in Table 3. Empiric use of combination therapy, carbapenems, glycopeptides, and antifungal agents were significantly more frequent in the de-escalation group. Among antifungal agents, echinocandins were minimally prescribed for empiric therapy (six patients in the de-escalation group with discontinuation in all but one case and one switch to azoles, one patient in the non-de-escalation group).
When taking into account the criteria for de-escalation, we found that 33 patients met three criteria (withdrawing, narrowing, and switching) in the de-escalation group but none of those who did not de-escalate. However, two criteria (withdrawing and narrowing) were reported in $17 \mathrm{pa}$ tients in the non-de-escalation group. No clinical change between days 0 and 3 allowed patients who were subsequently de-escalated to be differentiated from those who did not (Table 3).

Determinants of de-escalation in multivariate analysis were adequate empiric therapy (OR 9.60, 95 \% CI 4.0222.97, $p<0.001$ ), empiric use of vancomycin (OR 3.39, $95 \%$ CI 1.46-7.87, $p=0.004$ ), carbapenems (OR 2.64, $95 \%$ CI 1.01-6.91, $p=0.04$ ), and aminoglycosides (OR $2.31,95 \%$ CI 1.08-4.94, $p=0.03$ ), while presence of NFGNB (OR 0.28, $95 \%$ CI $0.09-0.89, p=0.03$ ) and presence of MDR bacteria (OR 0.21, $95 \%$ CI 0.09-0.52, $p<0.001)$ were the risk factors for non-de-escalation (c-index 0.880, 95 \% CI 0.832-0.928, Hosmer-Lemeshow test $p=0.14$ ) (Table 4).

At day 3, no difference was observed between patients with an unchanged regimen and those who underwent escalation, except for the higher proportions of 
Table 1 Demographic and clinical characteristics of the 206 patients with or without subsequent antibiotic de-escalation

\begin{tabular}{|c|c|c|c|c|}
\hline Characteristic & De-escalation $(n=110)$ & No de-escalation $(n=96)$ & Escalation $(n=65)$ & No change $(n=31)$ \\
\hline Male sex, $n(\%)$ & $61(55)$ & $56(58)$ & $35(54)$ & $21(68)$ \\
\hline Age, years, median (IQR) & $61(47-72)$ & $66(51-75)^{a}$ & $63(47-75)$ & $70(58-77)$ \\
\hline \multicolumn{5}{|l|}{ Comorbidities } \\
\hline Fatal underlying disease & $30(27)$ & $32(33)$ & $21(32)$ & $11(35)$ \\
\hline Cancer, $n(\%)$ & $37(34)$ & $36(38)$ & $23(35)$ & $13(42)$ \\
\hline Diabetes, $n(\%)$ & $17(15)$ & $15(16)$ & $9(14)$ & $6(19)$ \\
\hline Time since initial surgery, days, median (IQR) & $7(5-12)$ & $7(4-10)$ & $6(3-9)$ & $8(5-10)$ \\
\hline Antibiotic therapy before reoperation, $n(\%)$ & $73(66)$ & $68(71)$ & $47(72)$ & $21(68)$ \\
\hline Broad-spectrum interim antibiotic, $n$ (\%) & $34(31)$ & $37(39)$ & $27(42)$ & $10(32)$ \\
\hline \multicolumn{5}{|l|}{ Intraoperative diagnosis } \\
\hline Anastomotic leakage, $n(\%)$ & $45(41)$ & $27(28)$ & $19(29)$ & $8(26)$ \\
\hline Perforation or ischemia, $n(\%)$ & $33(30)$ & $36(38)$ & $20(31)$ & $16(52)^{\text {a\# }}$ \\
\hline Purulent collection, $n$ (\%) & $19(17)$ & $17(18)$ & $13(20)$ & $4(13)$ \\
\hline No cause, $n(\%)$ & $19(17)$ & $20(21)$ & $15(23)$ & $5(16)$ \\
\hline Contamination below transverse mesocolon, $n(\%)$ & $82(75)$ & $74(77)$ & $50(77)$ & $24(77)$ \\
\hline \multicolumn{5}{|l|}{ Characteristics at the time of ICU admission } \\
\hline Bacteremia, $n(\%)$ & $26(24)$ & $17(18)$ & $14(22)$ & $3(10)$ \\
\hline SAPS II score, median (IQR) & $45(34-54)$ & $47(35-57)$ & $44(34-56)$ & $51(42-61)^{\text {a\#t }}$ \\
\hline SOFA score, median (IQR) & $7(4-9)$ & $8(4-10)$ & $7(4-9)$ & $9(6-10)$ \\
\hline Hemodynamic failure ${ }^{\mathrm{b}}, n(\%)$ & $65(59)$ & $65(68)$ & $41(63)$ & $24(77)$ \\
\hline Respiratory failure ${ }^{b}, n(\%)$ & $54(49)$ & $40(42)$ & $26(40)$ & $14(45)$ \\
\hline Renal failure ${ }^{\mathrm{b}}, n(\%)$ & $21(19)$ & $18(19)$ & $13(20)$ & $5(16)$ \\
\hline
\end{tabular}

IQR interquartile range, SAPS // Simplified Acute Physiology Score II, SOFA Sequential Organ Failure Assessment

Patients without de-escalation were also analyzed in terms of subsequent antibiotic escalation or no change. Results are expressed as number and proportions or median (IQR)

${ }^{a} p<0.05$ versus de-escalation

bSOFA score of 3 or 4 for each organ

${ }^{\#} p<0.05$ versus escalation

aminoglycosides and antifungal therapy and the very low proportion adequate empiric therapy (Table 3 ).

\section{Clinical evaluation following de-escalation}

When comparing the patients who were de-escalated and those who did not de-escalate, we found no significant difference between days 3 and 7 (Table 5). In addition, no significant difference in morbidity or mortality criteria was observed between these two groups.

No significant differences in morbidity or mortality criteria were observed between patients who underwent escalation and those with an unchanged regimen, except for significantly increased proportions of surgical complications and reoperations in patients with an unchanged regimen. The risk factors for death at day 28 following surgery in a Cox model are presented in Table 6. The survival rate expressed by a Kaplan-Meier curve was similar between groups (log-rank test $p$ value 0.176) (Fig. 2).

The risk factors of in-hospital mortality on multivariate analysis were the emergency initial surgery (OR 2.81,
$95 \%$ CI $1.30-6.05, p=0.008)$ and SOFA score on admission (OR 1.42, 95 \% CI 1.24-1.62, $p<0.001$ ), while a decreased SOFA score at day 3 had a protective value (OR 0.14, 95 \% CI 0.06-0.31, $p<0.001$ ) (c-index 0.852, $95 \%$ CI 0.794-0.910, Hosmer-Lemeshow test $p=0.28$ ).

\section{Discussion}

In this single-center observational study, de-escalation was performed in $53 \%$ of patients treated for HCIAI. De-escalation concerned both antibacterial and antifungal therapies. The de-escalation procedure did not modify outcome. No initial clinical characteristic allowed identification of patients who were subsequently deescalated. The presence of MDR bacteria and NFGNB as well as initial monotherapy were the most relevant factors limiting de-escalation. No emergence of resistant organisms was observed following de-escalation in the patients who underwent subsequent reoperation.

In the absence of data in the literature, we consider that our results provide an encouraging perspective for antibiotic de-escalation in ICU patients with abdominal 


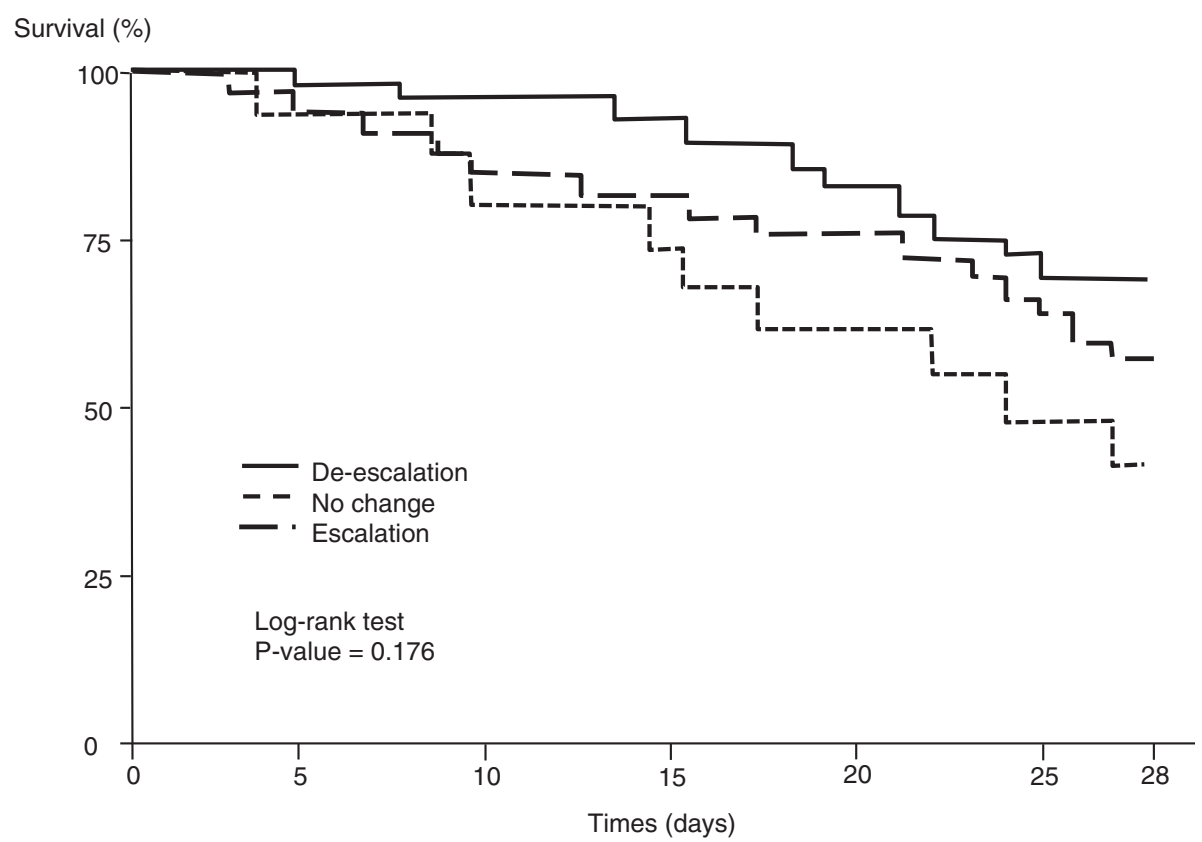

Fig. 2 Kaplan-Meier survival curves of patients with de-escalation, without any change, and with escalation

sepsis. However, some limitations should be considered. Only 110 (35\%) of 311 patients treated for HCIAI were de-escalated. This highly selected population and the inclusion and exclusion criteria could be considered to constitute a weakness, but they allow selection of cases in which de-escalation is possible. Our local policy for empiric and definitive antibiotic use and the local characteristics of microbial flora must be considered cautiously and cannot be generalized. The long study duration may also have led to changes in the case mix or in antibiotic susceptibility patterns over time, although our analysis did not confirm this hypothesis. Another major limitation in the interpretation of our results is the lack of assessment of the quality of source control. The exclusion of patients undergoing early reoperation probably limited the importance of this issue. The absence of consensual definitions for de-escalation is another issue to be considered. The quality of de-escalation could be considered incomplete in many patients in whom there is room for improvement, and further reduction of antibiotic use or the use of narrow-spectrum empiric therapy should be considered.

Table 2 Multidrug-resistant bacteria cultured from peritoneal fluid of patients with or without subsequent antibiotic de-escalation

\begin{tabular}{|c|c|c|c|c|}
\hline Microorganisms & De-escalation & No de-escalation & Escalation & No change \\
\hline Total number of multidrug-resistant bacteria, $n(\%)$ & $29(9)$ & $74(24)^{a}$ & $58(28)$ & $16(16)^{b}$ \\
\hline Gram-positive bacteria, $n$ (\%) & $15(5)$ & $39(13)^{a}$ & $33(16)$ & $6(6)^{b}$ \\
\hline Enterococci, $n(\%)$ & $3(1)$ & $9(3)$ & $7(3)$ & $2(2)$ \\
\hline Staphylococci, n (\%) & $12(4)$ & $29(9)$ & $25(12)$ & $4(4)$ \\
\hline Staphylococcus aureus, n (\%) & - & $8(3)$ & $6(3)$ & $2(2)$ \\
\hline Gram-negative bacteria, $n$ (\%) & $14(5)$ & $35(11)^{a}$ & $25(12)$ & $10(10)$ \\
\hline Enterobacteriaceae, n (\%) & $10(3)$ & $24(8)$ & $18(9)$ & $6(6)$ \\
\hline Escherichia coli, $n$ (\%) & $1(0)$ & $11(4)$ & $8(4)$ & $3(3)$ \\
\hline Enterobacter spp., $n(\%)$ & $5(2)$ & $9(3)$ & $8(4)$ & $1(1)$ \\
\hline Nonfermenting Gram-negative bacilli, $n$ (\%) & $3(1)$ & $11(4)$ & $7(3)$ & $4(4)$ \\
\hline Pseudomonas spp., $n$ (\%) & $2(1)$ & $6(2)$ & $4(2)$ & $2(2)$ \\
\hline Total number of cultured bacteria, $n$ & 307 & 311 & 210 & 101 \\
\hline
\end{tabular}

Among the 96 patients without de-escalation, the results were analyzed in terms of subsequent antibiotic escalation or no change ${ }^{a} p<0.01$ versus de-escalation

${ }^{\mathrm{b}} p<0.05$ versus escalation 
Table 3 Anti-infective regimens in patients with or without de-escalation and clinical characteristics at day 3

\begin{tabular}{|c|c|c|c|c|}
\hline & De-escalation $(n=110)$ & No de-escalation $(n=96)$ & Escalation $(n=65)$ & No change $(n=31)$ \\
\hline \multicolumn{5}{|l|}{ Empiric antibiotic therapy } \\
\hline Monotherapy, $n$ (\%) & $13(12)$ & $32(33)^{a}$ & $20(31)$ & $12(29)$ \\
\hline Combination of two drugs, $n(\%)$ & $40(36)$ & $34(35)$ & $26(40)$ & $8(26)$ \\
\hline Combination of three drugs or more, $n(\%)$ & 57 (49) & $30(33)^{b}$ & $19(29)$ & $11(35)$ \\
\hline Carbapenem, $n(\%)$ & $35(32)$ & $15(16)^{\mathrm{a}}$ & $10(15)$ & $5(16)$ \\
\hline Piperacillin-tazobactam, n (\%) & $67(61)$ & $60(63)$ & $40(62)$ & $20(65)$ \\
\hline Vancomycin, $n(\%)$ & $57(52)$ & $23(24)^{a}$ & $15(23)$ & $8(26)$ \\
\hline Aminoglycosides, $n(\%)$ & $59(54)$ & $33(34)^{\mathrm{a}}$ & $27(42)$ & $6(19)^{b}$ \\
\hline Fluoroquinolones, n (\%) & $6(5)$ & $11(11)$ & $10(15)$ & $1(3)$ \\
\hline Antifungal therapy, $n$ (\%) & $47(43)$ & $23(24)^{a}$ & $11(17)$ & $12(39)^{b}$ \\
\hline Azoles, $n(\%)$ & $41(37)$ & $20(21)^{a}$ & $9(14)$ & $11(35)^{b}$ \\
\hline Adequate empiric therapy, $n(\%)$ & $100(91)$ & $37(39)^{\mathrm{a}}$ & $9(14)$ & $28(90)^{c}$ \\
\hline \multicolumn{5}{|l|}{ Reevaluation of antibiotic therapy } \\
\hline Discontinuation of carbapenems ${ }^{d}, n(\%)$ & $27 / 35(77)$ & $4 / 15(27)$ & $4 / 10(40)$ & - \\
\hline Discontinuation of piperacillin-tazobactam ${ }^{d}, n(\%)$ & $50 / 67(75)$ & $25 / 60(42)$ & $25 / 40(63)$ & - \\
\hline Discontinuation of vancomycin ${ }^{d}, n(\%)$ & $46 / 57(81)$ & 6/23 (26) & $6 / 15(40)$ & - \\
\hline Discontinuation of aminoglycosides ${ }^{d}, n(\%)$ & $54 / 59(92)$ & $21 / 33(64)$ & $21 / 27(78)$ & - \\
\hline Discontinuation of fluoroquinolones ${ }^{\mathrm{d}}, n(\%)$ & $2 / 6(33)$ & $6 / 11(55)$ & $6 / 10(60)$ & - \\
\hline Discontinuation of antifungal agent ${ }^{d}, n(\%)$ & 23/47 (49) & $4 / 23(17)$ & 4/11 (36) & - \\
\hline Withdrawal of at least one agent, $n(\%)$ & $110(100)$ & $42(47)^{a}$ & $42(65)$ & - \\
\hline Narrowing spectrum, $n(\%)$ & $74(67)$ & $18(19)^{\mathrm{a}}$ & $18(28)$ & - \\
\hline Switch to monotherapy, $n$ (\%) & $54(49)$ & $7(7)^{a}$ & $7(11)$ & - \\
\hline Interruption of unnecessary agent, $n$ (\%) & $78(71)$ & $20(21)^{a}$ & $20(31)$ & - \\
\hline \multicolumn{5}{|l|}{ Clinical changes between days 0 and 3} \\
\hline Changes in SOFA score, median (IQR) & $-2(-4$ to -1$)$ & $-2(-4$ to 0$)$ & $-2(-4$ to 0$)$ & $-2(-3$ to 0$)$ \\
\hline Decreased SOFA score, $n(\%)$ & $69(63)$ & $57(59)$ & $38(58)$ & $19(61)$ \\
\hline Decreased temperature, $n(\%)$ & $69(63)$ & $64(67)$ & $41(63)$ & $23(74)$ \\
\hline Decreased WBC, $n(\%)$ & $38(35)$ & $32(33)$ & $23(35)$ & $9(29)$ \\
\hline Clinical improvement at day 3,n (\%) & $17(15)$ & $18(19)$ & $14(22)$ & $4(13)$ \\
\hline
\end{tabular}

IQR interquartile range, SOFA Sequential Organ Failure Assessment, WBC white blood cell count

Among those without de-escalation, the results were analyzed in terms of subsequent antibiotic escalation or no change. Results are expressed as number and proportion of the total number of patients

a $p<0.01$ versus de-escalation

${ }^{\mathrm{b}} p<0.05$ versus de-escalation

$c_{p}<0.01$ versus escalation therapy

${ }^{\mathrm{d}}$ Proportions are expressed as the number of discontinuations of the drug to the total number of patients empirically receiving this class of drug

Observational and retrospective studies have suggested that the de-escalation strategy is a safe approach in patients with severe sepsis or septic shock $[10-12,15,18$, $19,22,35]$. In recent European observational trials, the de-escalation rate ranged between $12.8 \%$ of cases in a multicenter study in 41 French ICUs [35] and $64 \%$ in a single-center analysis focused on patients with severe sepsis and septic shock [15]. Some prospective observational studies have suggested that mortality rates were at least not worse than those observed in patients not de-escalated [16, 36-38]. In these reports, the lengths of ICU and hospital stay were not significantly different $[36,37]$. Other authors have reported that deescalation therapy could even significantly improve the prognosis $[4,13,39]$.

Prospective randomized trials addressing the issue of de-escalation are extremely rare. In a cohort of 290 patients treated for VAP, Micek et al. reported a decreased duration of antibiotic therapy and no significant differences in terms of secondary episodes of VAP and hospital mortality [37]. In a group of 116 patients with severe sepsis either assigned or not to de-escalation, 
Table 4 Uni- and multivariate analyses of risk factors for de-escalation

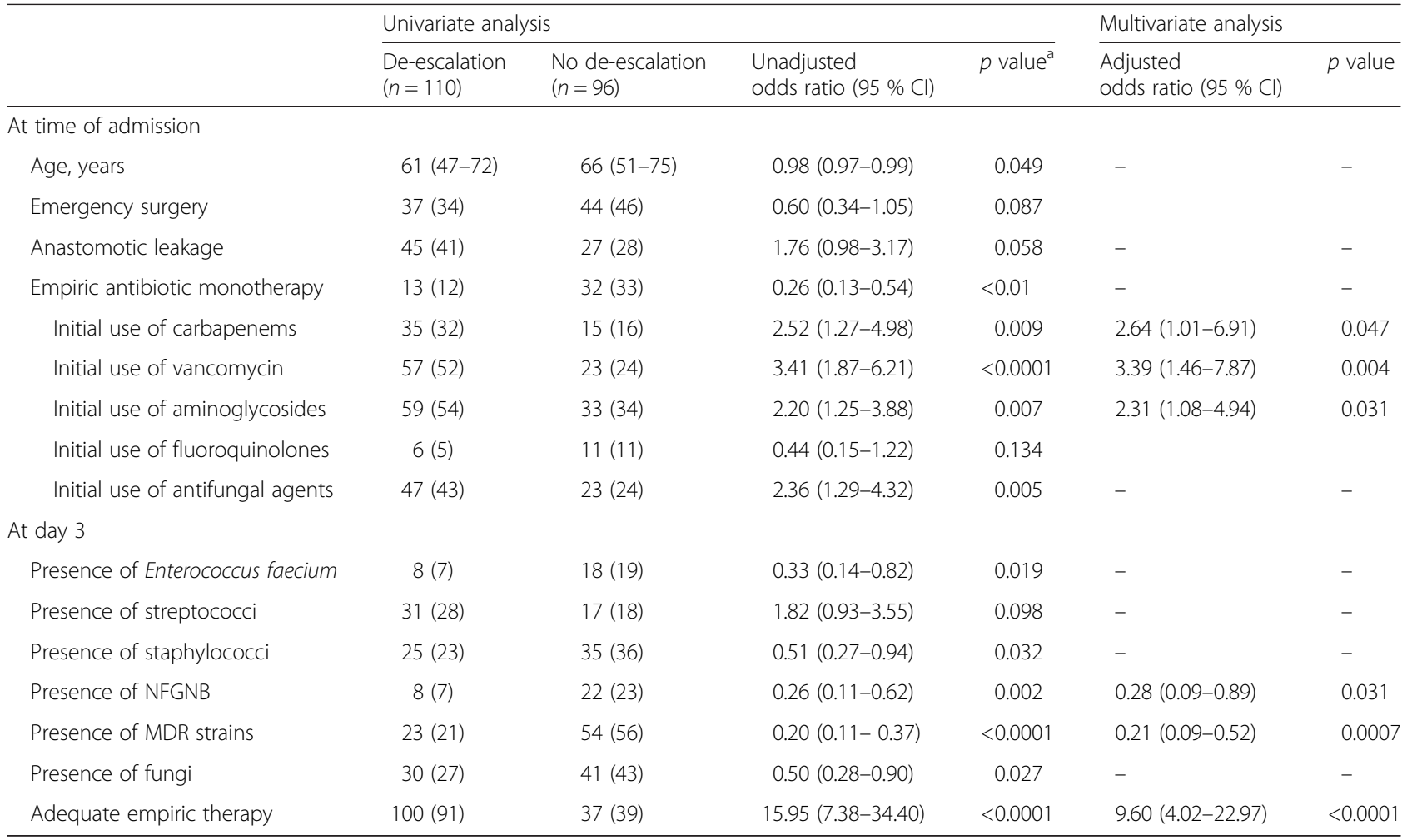

MDR multidrug-resistant, NFGNB nonfermenting Gram-negative bacilli

The c-index of the final model is $0.880(95 \% \mathrm{Cl} 0.832-0.928)$, and the Hosmer-Lemeshow test $p$ value is 0.14

${ }^{\mathrm{a}} p$ values are derived from Fisher's exact test or Wilcoxon test

Leone et al. demonstrated that de-escalation was inferior to continuation of the initial antibiotic therapy with length of stay as the primary outcome parameter [40]. Furthermore, antibiotic use was higher in the deescalation group with a higher number of superinfections in the de-escalation group, but mortality was similar in the two groups [40].

Only four retrospective, single-center, observational studies have described de-escalation practices for patients with peritonitis $[14,18,21,22]$, including between 113 and 229 patients, $10-38 \%$ of whom presented with peritonitis. Although the observed de-escalation rate was $23-58 \%$ in this population, none of these studies provided any information on the outcome of de-escalated patients. Our analysis is the first to focus on this surgical population, and our results suggest that de-escalation is safe and does not change the clinical outcome. On the basis of our results, a prospective multicenter study would appear to be feasible.

Obstacles to de-escalation have been clearly identified in the literature [20]. The lack of appropriate empiric therapy is the first point to be considered [13, 22]. A high rate of MDR bacteria is an obvious reason for inadequate empiric therapy and consequently a recognized factor for limited de-escalation [20]. However, although a recent analysis suggested that polymicrobial infection was a negative factor for de-escalation [20], we did not observe this trend in the patients in our present study. Narrow-spectrum empiric therapy is obviously another important determinant limiting the frequency of de-escalation [22]. However, narrow-spectrum empiric therapy is not an issue in intra-abdominal infections in which treatment should at least target anaerobes and Enterobacteriaceae [41]. On the contrary, monotherapy has been proposed for the treatment of peritonitis [41], and this policy could be a limitation on de-escalation [18].

Poor or absent clinical improvement is another factor limiting de-escalation. In the present study, clinical and laboratory parameters of day 3 were unable to differentiate patients in whom de-escalation would be feasible and those in whom de-escalation could not be performed. Interestingly, Garnacho-Montero et al. reported lower SOFA scores at the time of de-escalation [4], suggesting that the criteria for de-escalation may change from one population to another. This also means that prescribers should rely on microbiologic samples and the laboratory results more than any other criteria. The confidence of prescribers in their initial therapy is also based on two parameters that have been only minimally 
Table 5 Clinical presentation at day 7 of empiric therapy and outcome in patients with or without de-escalation

\begin{tabular}{|c|c|c|c|c|}
\hline & De-escalation $(n=110)$ & No de-escalation $(n=96)$ & Escalation $(n=65)$ & No change $(n=31)$ \\
\hline \multicolumn{5}{|l|}{ Definitive anti-infective therapy } \\
\hline Monotherapy, n (\%) & $64(58)$ & $21(22)$ & $9(14)$ & $12(29)^{a}$ \\
\hline Combination of two drugs, $n(\%)$ & $33(30)$ & $39(41)$ & $31(48)$ & $8(26)^{b}$ \\
\hline Combination of three drugs or more, $n(\%)$ & $13(12)$ & $36(38)$ & $25(38)$ & $11(35)$ \\
\hline Use of carbapenems, $n$ (\%) & $5(5)$ & $23(24)^{a}$ & $18(28)$ & $5(16)$ \\
\hline Use of piperacillin-tazobactam, $n$ (\%) & $28(25)$ & $39(41)^{\mathrm{b}}$ & $19(29)$ & $20(65)^{a}$ \\
\hline Use of vancomycin, $n$ (\%) & $11(10)$ & $41(43)^{a}$ & $33(51)$ & $8(26)^{b}$ \\
\hline Use of antifungals, $n(\%)$ & $29(26)$ & $43(45)^{\mathrm{a}}$ & $31(48)$ & $12(39)$ \\
\hline Use of azoles, $n(\%)$ & $28(25)$ & $39(41)^{\mathrm{b}}$ & $28(43)$ & $11(35)$ \\
\hline Use of echinocandins, $n$ (\%) & $1(1)$ & $3(3)$ & $3(5)$ & - \\
\hline Duration of anti-infective therapy, days, median (IQR) & $10(10-14)$ & $10(10-14)$ & $10(10-14)$ & $10(10-14)$ \\
\hline \multicolumn{5}{|l|}{ Clinical changes between days 3 and 7} \\
\hline Number of cases at day 7 & 91 & 75 & 51 & 24 \\
\hline Changes in SOFA score ${ }^{c}$, median (IQR) & $-1(-3$ to 0$)$ & $-2(-4$ to 0$)$ & $-2(-4$ to 0$)$ & $-2(-4$ to 0$)$ \\
\hline Decreased SOFA score ${ }^{c}, n(\%)$ & $57(63)$ & $48(64)$ & $35(54)$ & $13(42)$ \\
\hline Decreased temperature ${ }^{c}, n(\%)$ & $38(42)$ & $36(48)$ & $27(42)$ & $9(29)$ \\
\hline Decreased WBC ${ }^{C}, n(\%)$ & $31(34)$ & $17(23)$ & $12(18)$ & $5(16)$ \\
\hline Clinical improvement at day $7^{c}, n(\%)$ & $16(18)$ & $9(12)$ & $9(14)$ & - \\
\hline Discharge between days 3 and $7, n(\%)$ & $20(22)$ & $15(20)$ & $10(20)$ & $5(21)$ \\
\hline Death between days 3 and $7, n(\%)$ & $1(1)$ & $3(4)$ & $2(4)$ & $1(4)$ \\
\hline Medical complications & $12(12)$ & $14(16)$ & $6(10)$ & $8(29)$ \\
\hline Surgical complications & $26(25)$ & $21(24)$ & $10(17)$ & $11(39)^{b}$ \\
\hline Reoperation, $n(\%)$ & $38(35)$ & $35(36)$ & $19(29)$ & $16(51)^{\mathrm{b}}$ \\
\hline Time to reoperation, days, median (IQR) & $6(5-9)$ & $6(5-8)$ & $5(4-8)$ & $7(6-10)$ \\
\hline Superinfection on subsequent reoperation ${ }^{d}, n(\%)$ & $23(61)$ & $23(66)$ & $13(68)$ & $10(63)$ \\
\hline Emergence of MDR strains ${ }^{d}, n(\%)$ & $21(55)$ & $20(57)$ & $11(58)$ & $9(56)$ \\
\hline Emergence of ESBL Enterobacteriaceae ${ }^{\mathrm{d}}, n(\%)$ & $5(13)$ & $5(14)$ & $3(16)$ & $2(13)$ \\
\hline Emergence of MDR NFGNB ${ }^{d}, n(\%)$ & $9(24)$ & $5(14)$ & $2(11)$ & $3(19)$ \\
\hline Emergence of MRSA ${ }^{\mathrm{d}}, n(\%)$ & $9(24)$ & $8(23)$ & $6(32)$ & $2(13)$ \\
\hline Duration of mechanical ventilation ${ }^{\mathrm{e}}$, days, median (IQR) & $7(3-13)$ & $7(3-11)$ & $7(2-10)$ & $7(3-15)$ \\
\hline ICU length of staye, days, median (IQR) & $12(8-20)$ & $12(8-21)$ & $12(8-21)$ & $14(5-23)$ \\
\hline Survival at day $28, n(\%)$ & $91(83)$ & $72(75)$ & $51(78)$ & $21(68)$ \\
\hline ICU mortality rate, $n(\%)$ & $23(21)$ & $32(33)^{b}$ & $18(28)$ & $14(45)$ \\
\hline Hospital mortality rate, $n(\%)$ & $25(23)$ & $33(34)$ & $19(29)$ & $14(45)$ \\
\hline
\end{tabular}

ESBL extended-spectrum $\beta$-lactamase, ICU intensive care unit, IQR interquartile range, MDR multidrug-resistant, MRSA methicillin-resistant Staphylococcus aureus, NFGNB nonfermenting Gram-negative bacilli, SOFA Sequential Organ Failure Assessment, WBC white blood cell count

Among those without de-escalation, the results were analyzed in terms of subsequent antibiotic escalation or no change

${ }^{a} p<0.01$ versus escalation therapy

${ }^{\mathrm{b}} p<0.05$ versus escalation therapy

${ }^{c}$ Results expressed as number of patients at day 7 in the same group

${ }^{\mathrm{d}}$ Results expressed as number of patients who underwent reoperation in the same group

${ }^{\text {e}}$ Results calculated for ICU survivor patients

assessed in the literature: adequacy of source control and pharmacokinetics of anti-infective agents.

Several reports have indicated early improvement in patients who underwent de-escalation. Paskovaty et al., in a cohort of adult patients with cancer admitted to the
ICU for severe sepsis, reported a significantly decreased SOFA score on day 5 [42]. Two studies of patients with nosocomial pneumonia and ICU-acquired pneumonia reported early decreased SOFA and Acute Physiology and Chronic Health Evaluation II scores in the de- 
Table 6 Uni- and multivariate analyses of risk factors for 28-day mortality

\begin{tabular}{|c|c|c|c|c|c|c|}
\hline & \multicolumn{3}{|c|}{ Univariate analysis } & \multirow[b]{2}{*}{$p$ value $^{a}$} & \multicolumn{2}{|l|}{ Multivariate analysis } \\
\hline & $\begin{array}{l}\text { Death at day } \\
28(n=43)\end{array}$ & $\begin{array}{l}\text { Survival at day } \\
28(n=163)\end{array}$ & $\begin{array}{l}\text { Unadjusted hazard } \\
\text { ratio }(95 \% \mathrm{Cl})\end{array}$ & & $\begin{array}{l}\text { Adjusted hazard } \\
\text { ratio }(95 \% \mathrm{Cl})\end{array}$ & $p$ value \\
\hline Age, years & $69(56-78)$ & $62(46-72)$ & $1.022(1.003-1.042)$ & 0.023 & $1.034(1.011-1.059)$ & 0.004 \\
\hline Emergency surgery & $24(56)$ & $57(35)$ & $2.072(1.135-3.783)$ & 0.015 & - & - \\
\hline Surgery below the mesocolon & $28(65)$ & $128(79)$ & $0.561(0.299-1.050)$ & 0.075 & $0.427(0.215-0.848)$ & 0.015 \\
\hline SOFA score & $10(7-11)$ & $7(4-9)$ & $1.261(1.153-1.380)$ & $<0.0001$ & $1.291(1.168-1.427)$ & $<0.0001$ \\
\hline SAPS II score & $52(45-61)$ & $44(32-53)$ & $1.039(1.019-1.060)$ & $<0.0001$ & - & - \\
\hline Initial use of piperacillin-tazobactam & $22(51)$ & $105(64)$ & $0.636(0.350-1.157)$ & 0.117 & - & - \\
\hline Empiric antifungal therapy & $21(49)$ & $49(30)$ & $1.980(1.089-3.601)$ & 0.029 & - & - \\
\hline Other Enterobacteriaceae & $5(12)$ & $34(21)$ & $0.538(0.212-1.366)$ & 0.195 & $0.342(0.1219-0.961)$ & 0.0419 \\
\hline Presence of Candida spp. & $19(44)$ & $52(32)$ & $1.569(0.859-2.865)$ & 0.150 & $2.641(1.3471-5.179)$ & 0.0047 \\
\hline Decreased SOFA score at day 3 & $17(40)$ & $109(67)$ & $0.372(0.202-0.686)$ & 0.0015 & $0.311(0.1632-0.593)$ & 0.0004 \\
\hline Antibiotic strategy & & & & 0.189 & & \\
\hline De-escalation & $19(44)$ & $91(56)$ & $0.488(0.227-1.051)$ & & $0.566(0.2503-1.278)$ & 0.171 \\
\hline No change & $10(23)$ & $21(13)$ & Reference & & Reference & Reference \\
\hline Escalation & $14(33)$ & $51(31)$ & $0.627(0.278-1.411)$ & & $0.508(0.2154-1.198)$ & 0.122 \\
\hline
\end{tabular}

SAPS II Simplified Acute Physiology Score II, SOFA Sequential Organ Failure Assessment

${ }^{a} p$ values are from Fisher exact tests or Wilcoxon tests

bOther Enterobacteriaceae: analysis of all Enterobacteriaceae except Escherichia coli, Klebsiella spp., and Enterobacter spp.

escalation groups $[43,44]$. On the contrary, the incidence of organ failure at day 7 was similar in our patients with or without de-escalation. There is no obvious explanation for this discrepancy, but medical and surgical patients with sepsis may respond in different ways.

The rate of antibiotic escalation, although regularly discussed, is rarely assessed in the literature. In recent papers, this rate has ranged between $6.6 \%$ and $7.9 \%[18,22]$. However, Garnacho et al. reported escalation in $19 \%$ of patients despite adequate empiric therapy [4]. The mortality rate in this cohort was significantly increased compared with de-escalation or unchanged therapy (42.9\% versus $27.4 \%$ and $32.6 \%$, respectively; $p=0.006$ ) [4], while Gonzales et al. reported that escalation did not induce any significant change in prognosis [22]. In these two studies, the heterogeneous case mix resulted in complex analysis of these data. Few studies have reported the frequency and prognosis of escalation in peritonitis, but the effect of escalation appears to be less obvious in the present cohort.

Several beneficial effects of de-escalation have been hypothesized, including preservation of the patient's ecology and decreased emergence of MDR pathogens [3, 45]. However, these assumptions have never been clearly demonstrated. In the present study, we did not observe any significant change in the emergence of resistant pathogens in either the intra-abdominal site or extraabdominal sites following de-escalation. This is not surprising, as the detection of emerging MDR organisms was not a specific goal of this study and changes of gut microbiota of our patients were not targeted. Similarly, de-escalation does not change the overall duration of therapy. This point, already mentioned in other studies [22], was also observed in our present study.

An abundant literature exists regarding assessment of antibiotic de-escalation, but few data are available for antifungal agents. Several reports in candidemia or invasive candidiasis suggest that antifungal de-escalation is feasible [46-48], but no study has specifically addressed the issue of intra-abdominal infections. Unwarranted antifungal prescription is frequently reported in ICU patients, which raises both ecological and financial concerns [49]. Deescalation and/or discontinuation of antifungal treatments could be proposed more frequently. Our data suggest that antifungal de-escalation could be feasible with no specific complications.

\section{Conclusions}

De-escalation is a reasonable option, even in patients with polymicrobial infections such as HCIAI. However, MDR bacteria and NFGNB remain major obstacles to implementation of de-escalation. The prescriber must consider whether the determinants of success have been met, especially an adequate empiric therapy. Although our results are reassuring, this strategy needs to be confirmed in a multicenter, randomized, prospective trial. 


\section{Key messages}

- De-escalation is a reasonable option, even in polymicrobial infections such as health care-associated intra-abdominal infections.

- Multidrug-resistant bacteria and nonfermenting Gram-negative bacilli remain a major obstacle in de-escalation.

- The key determinants for de-escalation are susceptible microorganisms and adequate empiric therapy.

\section{Abbreviations}

ESBL: extended-spectrum $\beta$-lactamase; HCIAl: health care-associated intra-abdominal infection; ICU: intensive care unit; IQR: interquartile range; MDR: multidrug-resistant: MRSA: methicillin-resistant Staphylococcus aureus: NFGNB: nonfermenting Gram-negative bacilli; SAPS II: Simplified Acute Physiology Score II; SOFA: Sequential Organ Failure Assessment; VAP: ventilator-associated pneumonia; WBC: white blood cell count.

\section{Competing interests}

The authors declare that they have no competing interests.

\section{Authors' contributions}

PM, PA, NG, MD, NA, and JPM carried out the data acquisition, data processing, and data analysis. PM, PA, MD, and JG contributed to statistical analysis and participated in drafting the manuscript. All authors made substantial contributions to the conception and design of the study. All authors read and approved the final manuscript

\section{Author details}

'Département d'Anesthésie Réanimation, APHP, CHU Bichat-Claude Bernard, Paris, France. ${ }^{2}$ Université Denis Diderot, PRESS Sorbonne Cité, Paris, France. ${ }^{3}$ INSERM, UMR 1137, Infection, Antimicrobiens, Modélisation, Evolution, Paris, France. ${ }^{4}$ Laboratoire de Microbiologie, AP-HP, CHU Bichat-Claude Bernard, Paris, France. ${ }^{5}$ Service de Réanimation, Centre Hospitalier Sud Francilien, Corbeil-Essonnes, France. 'Service de Chirurgie Générale, APHP, CHU Bichat-Claude Bernard, Paris, France.

Received: 12 January 2016 Accepted: 16 March 2016 Published online: 07 April 2016

\section{References}

1. Weiss E, Zahar JR, Lesprit P, Ruppe E, Leone M, Chastre J, et al. Elaboration of a consensual definition of de-escalation allowing a ranking of $\beta$-lactams. Clin Microbiol Infect. 2015;21:649.e1-10.

2. Dellit TH, Owens RC, McGowan Jr JE, Gerding DN, Weinstein RA, Burke JP, et al. Infectious Diseases Society of America and the Society for Healthcare Epidemiology of America guidelines for developing an institutional program to enhance antimicrobial stewardship. Clin Infect Dis. 2007:44:159-77.

3. Dellinger RP, Levy MM, Rhodes A, Annane D, Gerlach H, Opal SM, et al. Surviving Sepsis Campaign: international guidelines for management of severe sepsis and septic shock, 2012. Intensive Care Med. 2013;39:165-228.

4. Garnacho-Montero J, Gutiérrez-Pizarraya A, Escoresca-Ortega A, Corcia-Palomo Y, Fernández-Delgado E, Herrera-Melero I, et al. De-escalation of empirical therapy is associated with lower mortality in patients with severe sepsis and septic shock. Intensive Care Med. 2014:40:32-40.

5. Giantsou E, Liratzopoulos N, Efraimidou E, Panopoulou M, Alepopoulou E, Kartali-Ktenidou S, et al. De-escalation therapy rates are significantly higher by bronchoalveolar lavage than by tracheal aspirate. Intensive Care Med. 2007;33:1533-40

6. Kollef MH. Hospital-acquired pneumonia and de-escalation of antimicrobial treatment. Crit Care Med. 2001;29:1473-5

7. Niederman MS. De-escalation therapy in ventilator-associated pneumonia. Curr Opin Crit Care. 2006;12:452-7.

8. Kollef $\mathrm{MH}$. What can be expected from antimicrobial de-escalation in the critically ill? Intensive Care Med. 2014;40:92-5.
9. Alvarez-Lerma F, Alvarez B, Luque P, Ruiz F, Dominguez-Roldan JM,

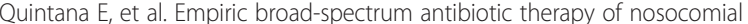
pneumonia in the intensive care unit: a prospective observational study. Crit Care. 2006:10:R78.

10. Eachempati SR, Hydo LJ, Shou J, Barie PS. Does de-escalation of antibiotic therapy for ventilator-associated pneumonia affect the likelihood of recurrent pneumonia or mortality in critically ill surgical patients? J Trauma. 2009;66:1343-8

11. Canadian Critical Care Trials Group. A randomized trial of diagnostic techniques for ventilator-associated pneumonia. N Engl J Med. 2006;355:2619-30.

12. Leone M, Garcin F, Bouvenot J, Boyadjev I, Visintini P, Albanese J, et al. Ventilator-associated pneumonia: breaking the vicious circle of antibiotic overuse. Crit Care Med. 2007:35:379-85.

13. Rello J, Vidaur L, Sandiumenge A, Rodriguez A, Gualis B, Boque C, et al. De-escalation therapy in ventilator-associated pneumonia. Crit Care Med. 2004;32:2183-90.

14. Heenen S, Jacobs F, Vincent JL. Antibiotic strategies in severe nosocomial sepsis: why do we not de-escalate more often? Crit Care Med. 2012:40:1404-9.

15. Leone M, Bourgoin A, Cambon S, Dubuc M, Albanese J, Martin C. Empirical antimicrobial therapy of septic shock patients: adequacy and impact on the outcome. Crit Care Med. 2003;31:462-7.

16. Mokart D, Slehofer G, Lambert J, Sannini A, Chow-Chine L, Brun JP, et al. De-escalation of antimicrobial treatment in neutropenic patients with severe sepsis: results from an observational study. Intensive Care Med. 2014:40:41-9.

17. Montravers P, Mira JP, Gangneux JP, Leroy O, Lortholary O. A multicentre study of antifungal strategies and outcome of Candida spp. peritonitis in intensive-care units. Clin Microbiol Infect. 2011;17:1061-7.

18. Morel J, Casoetto J, Jospe R, Aubert G, Terrana R, Dumont A, et al. De-escalation as part of a global strategy of empiric antibiotherapy management: a retrospective study in a medico-surgical intensive care unit. Crit Care. 2010;14:R225

19. Warren MM, Gibb AP, Walsh TS. Antibiotic prescription practice in an intensive care unit using twice-weekly collection of screening specimens: a prospective audit in a large UK teaching hospital. J Hosp Infect. 2005:59:90-5

20. Tabah A, Cotta MO, Garnacho-Montero J, Schouten J, Roberts JA, Lipman J, et al. A systematic review of the definitions, determinants, and clinical outcomes of antimicrobial de-escalation in the intensive care unit. Clin Infect Dis. 2016;62:1009-17.

21. De Waele JJ, Ravyts M, Depuydt P, Blot SI, Decruyenaere J, Vogelaers D. De-escalation after empirical meropenem treatment in the intensive care unit: fiction or reality? J Crit Care. 2010;25:641-6.

22. Gonzalez L, Cravoisy A, Barraud D, Conrad M, Nace L, Lemarié J, et al. Factors influencing the implementation of antibiotic de-escalation and impact of this strategy in critically ill patients. Crit Care. 2013;17:R140.

23. Augustin P, Kermarrec N, Muller-Serieys C, Lasocki S, Chosidow D, Marmuse $J P$, et al. Risk factors for multidrug resistant bacteria and optimization of empirical antibiotic therapy in postoperative peritonitis. Crit Care. 2010;14:R20

24. Montravers P, Dufour G, Guglieminotti J, Desmard M, Muller C, Houissa H, et al. Dynamic changes of microbial flora and therapeutic consequences in persistent intra-abdominal sepsis. Crit Care. 2015;19:70.

25. Montravers $\mathrm{P}$, Guglielminotti J, Zappella N, Desmard M, Muller C, Fournier $\mathrm{P}$ et al. Clinical features and outcome of postoperative peritonitis following bariatric surgery. Obes Surg. 2013;23:1536-44.

26. Marshall JC, Maier RV, Jimenez M, Dellinger EP. Source control in the management of severe sepsis and septic shock: an evidence-based review. Crit Care Med. 2004;32(11 Suppl):S513-26.

27. Comité de l'Antibiogramme de la Société Française de Microbiologie (CASFM) 2011. Recommandations 2011. Paris: Société Française de Microbiologie; 2011. http://www.sfm-microbiologie.org/UserFiles/files/casfm/ casfm 2011.pdf. Accessed 4 December 2015.

28. Magiorakos AP, Srinivasan A, Carey RB, Carmeli Y, Falagas ME, Giske CG, et al. Multidrug-resistant, extensively drug-resistant and pandrug-resistant bacteria: an international expert proposal for interim standard definitions for acquired resistance. Clin Microbiol Infect. 2012;18:268-81.

29. Dupont H, Bourichon A, Paugam-Burtz C, Mantz J, Desmonts JM. Can yeast isolation in peritoneal fluid be predicted in intensive care unit patients with peritonitis? Crit Care Med. 2003;31:752-7. 
30. Le Gall JR, Lemeshow S, Saulnier F. A new Simplified Acute Physiology Score (SAPS II) based on a European/North American multicenter study. JAMA. 2003;270:2957-63.

31. Vincent JL, Moreno R, Takala J, Willatts S, De Mendonça A, Bruining H, et al, The SOFA (Sepsis-related Organ Failure Assessment) score to describe organ dysfunction/failure. Intensive Care Med. 1996;22:707-10.

32. McCabe WR, Jackson GG. Gram-negative bacteremia. 1. Etiology and ecology. Arch Intern Med. 1962;110:847-55.

33. Montravers P, Gauzit R, Muller C, Marmuse JP, Fichelle A, Desmonts JM. Emergence of antibiotic-resistant bacteria in cases of peritonitis after intraabdominal surgery affects the efficacy of empirical antimicrobial therapy. Clin Infect Dis. 1996;23:486-94.

34. Paugam-Burtz C, Dupont H, Marmuse JP, Chosidow D, Malek L, Desmonts JM et al. Daily organ-system failure for diagnosis of persistent intra-abdominal sepsis after postoperative peritonitis. Intensive Care Med. 2002;28:594-8.

35. Montravers P, Dupont P, Gauzit R, Veber B, Bedos JP, Lepape A, et al. Strategies of initiation and streamlining of antibiotic therapy in 41 French intensive care units. Crit Care. 2010;15:R17.

36. Ibrahim EH, Ward S, Sherman G, Schaiff R, Fraser VJ, Kollef MH. Experience with a clinical guideline for the treatment of ventilator-associated pneumonia. Crit Care Med. 2001;29:1109-15.

37. Micek ST, Ward S, Fraser VJ, Kollef MH. A randomized controlled trial of an antibiotic discontinuation policy for clinically suspected ventilator-associated pneumonia. Chest. 2004;125:1791-9.

38. Soo Hoo GW, Wen YE, Nguyen TV, Goetz MB. Impact of clinical guidelines in the management of severe hospital-acquired pneumonia. Chest. 2005;128:2778-87

39. Kollef MH, Morrow LE, Niederman MS, Leeper KV, Anzueto A, Benz-Scott L, et al. Clinical characteristics and treatment patterns among patients with ventilator-associated pneumonia. Chest. 2006;129:1210-8.

40. Leone M, Bechis C, Baumstarck K, Lefrant JY, Albanese J, Jaber S, et al. De-escalation versus continuation of empirical antimicrobial treatment in severe sepsis: a multicenter non-blinded randomized noninferiority trial. Intensive Care Med. 2014;40:1399-408.

41. Solomkin JS, Mazuski JE, Bradley JS, Rodvold KA, Goldstein EJ, Baron EJ, et al. Diagnosis and management of complicated intra-abdominal infection in adults and children: guidelines by the Surgical Infection Society and the Infectious Diseases Society of America. Clin Infect Dis. 2010;50:133-64.

42. Paskovaty A, Pastores SM, Gedrimaite Z, Kostelecky N, Riedel ER, Seo SK Antimicrobial de-escalation in septic cancer patients: is it safe to back down? Intensive Care Med. 2015;41:2022-3.

43. Knaak E, Cavalieri SJ, Elsasser GN, Preheim LC, Gonitzke A, Destache CJ. Does antibiotic de-escalation for nosocomial pneumonia impact intensive care unit length of stay? Infect Dis Clin Pract. 2013:21:172-6.

44. Joung MK, Lee JA, Moon SY, Cheong HS, Joo EJ, Ha YE, et al. Impact of de-escalation therapy on clinical outcomes for intensive care unit-acquired pneumonia. Crit Care. 2011;15:R79.

45. Timsit JF, Harbarth S, Carlet J. De-escalation as a potential way of reducing antibiotic use and antimicrobial resistance in ICU. Intensive Care Med. 2014:40:1580-2.

46. Ruhnke M. Antifungal stewardship in invasive Candida infections. Clin Microbiol Infect. 2014;20 Suppl 6:11-8

47. Bal AM, Shankland GS, Scott G, Imtiaz T, Macaulay R, McGill M. Antifungal step-down therapy based on hospital intravenous to oral switch policy and susceptibility testing in adult patients with candidaemia: a single centre experience. Int J Clin Pract. 2014;68:20-7.

48. Bailly S, Leroy O, Montravers P, Constantin JM, Dupont H, Guillemot D, et al. Antifungal de-escalation was not associated with adverse outcome in critically ill patients treated for invasive candidiasis: post hoc analyses of the AmarCAND2 study data. Intensive Care Med. 2015;41:1931-40.

49. Azoulay E, Dupont H, Tabah A, Lortholary O, Stahl JP, Francais A, et al. Systemic antifungal therapy in critically ill patients without invasive fungal infection. Crit Care Med. 2012:40:813-22.

\section{Submit your next manuscript to BioMed Central and we will help you at every step:}

- We accept pre-submission inquiries

- Our selector tool helps you to find the most relevant journal

- We provide round the clock customer support

- Convenient online submission

- Thorough peer review

- Inclusion in PubMed and all major indexing services

- Maximum visibility for your research

Submit your manuscript at www.biomedcentral.com/submit

) Biomed Central 